\title{
DESINGULARIZATION BY BLOWINGS-UP AVOIDING SIMPLE NORMAL CROSSINGS
}

\author{
EDWARD BIERSTONE, SERGIO DA SILVA, PIERRE D. MILMAN, \\ AND FRANKLIN VERA PACHECO \\ (Communicated by Lev Borisov)
}

\begin{abstract}
It is shown that, for any reduced algebraic variety in characteristic zero, one can resolve all but simple normal crossings (snc) singularities by a finite sequence of blowings-up with smooth centres which, at every step, avoids points where the transformed variety together with the exceptional divisor has only snc singularities. The proof follows the philosophy of Bierstone and Milman's Resolution except for minimal singularities I (2012) that the desingularization invariant can be used together with natural geometric information to compute local normal forms of singularities.
\end{abstract}

\section{INTRODUCTION}

The subject of this article is partial resolution of singularities. The main result asserts that, for any (reduced) algebraic variety $X$ in characteristic zero, we can resolve all but simple normal crossings singularities by a finite sequence of blowingsup with smooth centres which, at every step, avoids points where the corresponding transform of $X$ together with the exceptional divisor has only simple normal crossings singularities. For background and motivation of the problem, see [3, 4 and [5]. Our proof follows the philosophy of [3] that the desingularization invariant of [1], 2] can be used together with natural geometric information to compute local normal forms of singularities.

Every algebraic variety can be embedded locally in an affine space. An algebraic variety $X$ has simple normal crossings (snc) at a point $a$ if, locally at $a$, there is an embedding $X \hookrightarrow Z$ (where $Z$ is smooth) and a regular coordinate system $\left(x_{1}, \ldots, x_{n}\right)$ for $Z$ at $a$ in which each irreducible component of $X$ is a coordinate hypersurface $\left(x_{i}=0\right)$, for some $i$. Thus, snc singularities are singularities of $h y$ persurfaces. ( $X$ is a hypersurface if, locally, $X$ can be defined by a principal ideal sheaf on a smooth variety.)

Our main problem can be reduced to the case that $X$ is a closed hypersurface in a smooth variety $Z$, essentially because the desingularization algorithm of [1, 2] blows up non-hypersurface points first. (A detailed argument can be found in

Received by the editors July 18, 2012 and, in revised form, February 4, 2013.

2010 Mathematics Subject Classification. Primary 14E15, 14J17, 32S45; Secondary 14B05, $32 \mathrm{~S} 05,32 \mathrm{~S} 10$.

Key words and phrases. Resolution of singularities, simple normal crossings, desingularization invariant.

Research supported in part by NSERC grants OGP0009070, MRS342058, USRA191085, CGS145594585 and OGP0008949. 
[3, 4].) We therefore state and prove the main result here only in the case of an embedded hypersurface (Theorem 1.4).

Definition 1.1. Let $X \hookrightarrow Z$ denote an embedded hypersurface ( $Z$ smooth) and let $E$ denote a divisor on $Z$. We say that $(X, E)$ has (or is) simple normal crossings (snc) at a point $a \in Z$ if $Z$ admits a regular system of coordinates $\left(x_{1}, \ldots, x_{p}, y_{1}, \ldots, y_{q}\right)$ (where $p \geq 0$ ) at $a$, in which the irreducible components of $X$ at $a$ are $\left(x_{i}=0\right), i=1, \ldots, p$, and the support of each component of $E$ at $a$ is $\left(y_{j}=0\right)$, for some $j$. We also say that $X$ (respectively, $E$ ) is snc at $a$ if $(X, \emptyset)$ (respectively, $(\emptyset, E))$ is snc at $a$. The pair $(X, E)$ is snc if it is snc at every point.

Consider $X \hookrightarrow Z$ and $E$ as in Definition 1.1. We consider the support of $E$ as a divisor $\sum_{j=1}^{r} H_{j}$ (where each component $H_{j}$ is a closed hypersurface in $Z$, not necessarily irreducible), or, equivalently, as the collection $\left\{H_{1}, \ldots, H_{r}\right\}$. The results in this article involve only the support of $E$, so we will write $E=\sum_{j=1}^{r} H_{j}$ or $E=\left\{H_{1}, \ldots, H_{r}\right\}$, by abuse of notation.

Notation 1.2. Given a sequence of blowings-up

$$
Z=Z_{0} \stackrel{\sigma_{1}}{\longleftarrow} Z_{1} \longleftarrow \cdots \stackrel{\sigma_{t}}{\longleftarrow} Z_{t}
$$

where each $\sigma_{j+1}$ has smooth centre $C_{j} \subset Z_{j}$, we write $X_{0}=X, E_{0}=E$, and, for each $j=0,1, \ldots$, we set

$X_{j+1}:=$ strict transform of $X_{j}$,

$E_{j+1}:=$ collection of strict transforms of the components of $E_{j}$ together with the exceptional divisor $\sigma_{j+1}^{-1}\left(C_{j}\right)$ of $\sigma_{j+1}$.

This notation will be used throughout the article.

Definition 1.3. A blowings-up $\sigma: Z^{\prime} \rightarrow Z$ is admissible (for $(X, E)$ ) if the centre $C$ of $\sigma$ is smooth, $C \subset X$ and $C$ is snc with respect to $E$ (where the latter means that, locally in regular coordinates, $C$ is a coordinate subspace and the support of each component of $E$ is a coordinate hypersurface; note that $C$ may lie in certain components of $E$ ). The sequence of blowings-up (1.1) is called admissible if each $\sigma_{j+1}$ is admissible for $\left(X_{j}, E_{j}\right)$.

If $E_{j}$ is snc and $\sigma_{j+1}$ is admissible, then $E_{j+1}$ is snc.

Theorem 1.4. Let $X \hookrightarrow Z$ denote an embedded hypersurface in characteristic zero and let $E$ denote a snc divisor on $Z$. Then there is a sequence of admissible blowings-up (1.1) such that

(1) $\left(X_{t}, E_{t}\right)$ has only snc singularities,

(2) each $\sigma_{j+1}$ is an isomorphism over the locus of snc points of $\left(X_{j}, E_{j}\right)$.

Moreover, the association of the desingularization sequence (1.1) to $(X, E)$ is functorial with respect to smooth morphisms that preserve the number of irreducible components of $X$ at every point.

Condition (2) of the theorem implies that each centre $C_{j} \subset X_{j}$.

See [1, Section 12] for an earlier approach to Theorem 1.4. Weaker versions of Theorem 1.4, where (2) is replaced by the condition that the morphism $\sigma_{t} \circ \cdots \circ \sigma_{1}$ is an isomorphism over the snc locus of $(X, E)$, can also be found in [6], [5] and [3. Thm. 3.4] (the latter is functorial as in Theorem 1.4). Our proof of Theorem 1.4 uses the desingularization invariant inv $=\operatorname{inv}_{(X, E)}$, in the spirit of [3]. Theorem 1.4 
is an important special case of the main theorem of 4$]$ and is used in the proof of the latter (see [4, Rmk. 1.3(4)]).

We refer to the Appendix of [3], Crash course on the desingularization invariant, for the definition of inv and how to compute it (see also [1, 2]), though some of the ideas will be recalled in Section 2 below. The invariant inv is defined iteratively over a sequence of blowings-up (1.1) which are inv-admissible (where the latter means that the successive blowings-up are admissible and inv is constant on each component of a centre).

A key ingredient in our proof of Theorem 1.4 is a characterization of snc (in any year of the desingularization history (1.1)) in terms of certain special values $\operatorname{inv}_{p, s}$ of inv (Definition 2.1). The blowings-up in Theorem 1.4 are obtained by following the standard desingularization algorithm [1, 2] until the maximum value of inv becomes one of the special values $\operatorname{inv}_{p, s}$. When inv attains a value $\operatorname{inv}_{p, s}$ at a point $a$, we can write a local normal form for the singularity (Lemma 3.3) and then simplify the normal form by a sequence of invariantly defined cleaning blowings-up (cf. [3, Sect.2]) until the singularity becomes snc. See [3, §1.2] for a motivation of the idea of cleaning. Cleaning blowings-up are admissible but not, in general, inv-admissible. Nevertheless, a modified version of inv can be defined over the particular cleaning sequences needed in this article (Section 4). This is enough to be able to repeat the preceding process on the complement of the snc locus until finally we resolve all but snc singularities.

Section 2 includes an example that illustrates the difference between the algorithm of Theorem 1.4 and the weaker version of [3, Thm. 3.4] or the standard desingularization algorithm of $[1]$.

\section{THE DESINGULARIZATION INVARIANT AND EXAMPLES}

The Crash course on the desingularization invariant [3, Section 5] is a prerequisite for our proof of Theorem 1.4, and it should be consulted for the notions of maximal contact, coefficient ideal, companion ideal, etc. We will recall a few of the ideas involved in order to fix notation.

Resolution of singularities in characteristic zero can be realized essentially by choosing, as each successive centre of blowing up, the locus of maximal values of the desingularization invariant inv $=\operatorname{inv}_{(X, E)}$ [1, 2]. The invariant inv is defined iteratively over a sequence of inv-admissible blowings-up (1.1). In particular, if $a \in Z_{j}$, then $\operatorname{inv}(a)$ depends on the previous blowings-up.

Let $a \in Z_{j}$. Then $\operatorname{inv}(a)$ has the form

$$
\operatorname{inv}(a)=\left(\nu_{1}(a), s_{1}(a), \ldots, \nu_{q}(a), s_{q}(a), \nu_{q+1}(a)\right),
$$

where $\nu_{k}(a)$ is a positive rational number if $k \leq q$, each $s_{k}(a)$ is a non-negative integer, and $\nu_{q+1}(a)$ is either 0 (the order of the unit ideal) or $\infty$ (the order of the zero ideal). The first entry $\nu_{1}(a)$ is the order $\operatorname{ord}_{a} X_{j}$ of $X_{j}$ at $a$. If $a \in Z_{0}$ (i.e., in "year zero"), $s_{1}(a)$ is the number of components of $E$ at $a$. The successive pairs $\left(\nu_{k}(a), s_{k}(a)\right)$ are defined inductively over maximal contact subvarieties of increasing codimension. $\operatorname{inv}(a)=(0)$ if and only if $a \in Z_{j} \backslash X_{j}$.

We order finite sequences of the form (2.1) lexicographically. Then $\operatorname{inv}(\cdot)$ is upper-semicontinuous on each $Z_{j}$, and infinitesimally upper-semicontinuous; i.e., if $a \in Z_{j}$, then $\operatorname{inv}(\cdot) \leq \operatorname{inv}(a)$ on $\sigma_{j+1}^{-1}(a)$. 
We also introduce truncations of inv. Let $\operatorname{inv}_{k+1}(a)$ denote the truncation of $\operatorname{inv}(a)$ after $s_{k+1}(a)$ (i.e., after the $(k+1)$ st pair), and let $\operatorname{inv}_{k+1 / 2}(a)$ denote the truncation of $\operatorname{inv}(a)$ after $\nu_{k+1}(a)$.

Given $a \in Z_{j}$, let $a_{i}$ denote the image of $a$ in $Z_{i}, i \leq j$. (We will speak of year $i$ in the history of blowings-up.) The year of birth of $\operatorname{inv}_{k+1 / 2}(a)$ (or $\operatorname{inv}_{k+1}(a)$ ) denotes the smallest $i$ such that $\operatorname{inv}_{k+1 / 2}(a)=\operatorname{inv}_{k+1 / 2}\left(a_{i}\right)$ (respectively, $\left.\operatorname{inv}_{k+1}(a)=\operatorname{inv}_{k+1}\left(a_{i}\right)\right)$.

Let $a \in Z_{j}$. Let $E(a)$ denote the set of components of $E_{j}$ which pass through $a$. The entries $s_{k}(a)$ of $\operatorname{inv}(a)$ are the sizes of certain subblocks of $E(a)$ : Let $i$ denote the birth-year of $\operatorname{inv}_{1 / 2}(a)=\nu_{1}(a)$, and let $E^{1}(a)$ denote the collection of elements of $E(a)$ that are strict transforms of components of $E_{i}$ (i.e., strict transforms of elements of $\left.E\left(a_{i}\right)\right)$. Set $s_{1}(a):=\# E^{1}(a)$. We define $s_{k+1}(a)$, in general, by induction on $k$ : Let $i$ denote the year of birth of $\operatorname{inv}_{k+1 / 2}(a)$ and let $E^{k+1}(a)$ denote the set of elements of $E(a) \backslash\left(E^{1}(a) \cup \cdots \cup E^{k}(a)\right)$ that are strict transforms of components of $E_{i}$. Set $s_{k+1}(a):=\# E^{k+1}(a)$. Relative to $\operatorname{inv}_{k+1 / 2}(a)$, the elements of $E^{k+1}(a)$ are "old" components of the exceptional divisor, and $\mathcal{E}^{k+1}(a):=E(a) \backslash\left(E^{1}(a) \cup \cdots \cup E^{k+1}(a)\right)$ is the set of "new" components.

The successive pairs in $\operatorname{inv}(a)$ are calculated using "marked ideals" — collections of data that are computed iteratively on "maximal contact" subspaces of increasing codimension. A marked ideal $\underline{\mathcal{I}}$ is a quintuple $\underline{\mathcal{I}}=(Z, N, E, \mathcal{I}, d)$, where $Z \supset N$ are smooth varieties, $E=\sum_{i=1}^{s} H_{i}$ is a simple normal crossings divisor on $Z$ which is transverse to $N, \mathcal{I} \subset \mathcal{O}_{N}$ is an ideal, and $d \in \mathbb{N}$. (See [3, Defns. 5.5].)

The cosupport of $\underline{\mathcal{I}}, \operatorname{cosupp} \underline{\mathcal{I}}:=\left\{x \in N: \operatorname{ord}_{x} \mathcal{I} \geq d\right\}$. We say that $\underline{\mathcal{I}}$ is of maximal order if $d=\max \left\{\operatorname{ord}_{x} \mathcal{I}: x \in \operatorname{cosupp} \underline{\mathcal{I}}\right\}$.

A blowing-up $\sigma: Z^{\prime} \rightarrow Z$ (with smooth centre $C$ ) is $\mathcal{I}$-admissible (or simply admissible) if $C \subset \operatorname{cosupp} \underline{\mathcal{I}}$, and $C, E$ have only normal crossings. The (controlled) transform of $\mathcal{I}$ by an admissible blowing-up $\sigma: Z^{\prime} \rightarrow Z$ is the marked ideal $\underline{\mathcal{I}}^{\prime}=\left(Z^{\prime}, N^{\prime}, E^{\prime}, \mathcal{I}^{\prime}, d^{\prime}=d\right)$, where $N^{\prime}$ is the strict transform of $N$ by $\sigma$, $E^{\prime}=\sum_{i=1}^{s+1} H_{i}^{\prime}$ (where $H_{i}^{\prime}$ is the strict transform of $H_{i}$, for each $i=1, \ldots, s$, and $H_{s+1}^{\prime}$ is the exceptional divisor $\sigma^{-1}(C)$ of $\sigma$ ), $\mathcal{I}^{\prime}:=\mathcal{I}_{\sigma^{-1}(C)}^{-d} \cdot \sigma^{*}(\mathcal{I}$ ) (where $\mathcal{I}_{\sigma^{-1}(C)} \subset \mathcal{O}_{N^{\prime}}$ denotes the ideal of $\left.\sigma^{-1}(C)\right)$. In this definition, note that $\sigma^{*}(\mathcal{I})$ is divisible by $\mathcal{I}_{\sigma^{-1}(C)}^{d}$ and $E^{\prime}$ is a normal crossings divisor transverse to $N^{\prime}$, because $\sigma$ is admissible.

Computation of inv and of the centre of blowing up begins, in year zero, with the marked ideal $\underline{\mathcal{I}}^{0}=\underline{\mathcal{I}}_{X}:=\left(Z, Z, E, \mathcal{I}_{X}, 1\right)$, where $\mathcal{I}_{X} \subset \mathcal{O}_{Z}$ is the ideal of $X$. Consider a point $a \in X_{j}$, in an arbitrary year $j$. We will simplify notation by writing $Z, X, E$, etc., instead of $Z_{j}, X_{j}, E_{j}$, etc., when the year is understood. At $a$, the computation begins with $\underline{\mathcal{I}}^{0}=\left(Z, Z, \mathcal{E}, \mathcal{I}^{0}, 1\right)$, restricted to some neighbourhood $U$ of $a$, where $\mathcal{E}=\mathcal{E}(a)=E(a)$ and $\mathcal{I}^{0}$ denotes the transform of $\mathcal{I}_{X_{0}}$ (iterating the definition above). Then $\mathcal{I}^{0}$ equals the product of $\mathcal{I}_{X}=\mathcal{I}_{X_{j}}$ and a monomial in generators of the ideals of the elements of $\mathcal{E}$.

We pass from $\underline{\mathcal{I}}^{0}$ to the companion ideal $\underline{\mathcal{J}}^{0}=\underline{\mathcal{G}}\left(\underline{\mathcal{I}}^{0}\right)$, which is obtained from the product decomposition $\mathcal{I}^{0}=\mathcal{M}\left(\underline{\mathcal{I}}^{0}\right) \cdot \mathcal{R}\left(\underline{\mathcal{I}}^{0}\right)$, where $\mathcal{M}\left(\underline{\mathcal{I}}^{0}\right)$ is the monomial part of $\mathcal{I}^{0}$ (it is a monomial in exceptional divisors) and $\mathcal{R}\left(\underline{\mathcal{I}}^{0}\right)$ is the residual part of $\mathcal{I}^{0}$ (divisible by no exceptional divisor). See [3, $\S \S 5.6,5.8]$. Here, clearly $\mathcal{R}\left(\underline{\mathcal{I}}^{0}\right)=\mathcal{I}_{X}$ and, since the associated multiplicity of $\underline{\mathcal{I}}^{0}$ is $1, \underline{\mathcal{J}}^{0}=\left(\mathcal{E}, \mathcal{R}\left(\underline{\mathcal{I}}^{0}\right), d\right)=\left(\mathcal{E}, \overline{\mathcal{I}}_{X}, d\right):=$ $\left(Z, Z, \mathcal{E}, \mathcal{I}_{X}, d\right)$, where $d=\operatorname{ord}_{a} \mathcal{I}_{X}$. We set $\operatorname{inv}_{1 / 2}(a)=\nu_{1}(a)=d$. 
Since $\mathcal{J}^{0}$ is of maximal order, we can pass to the coefficient ideal plus boundary $\underline{\mathcal{I}}^{1}=\left(Z, \bar{N}^{1}, \mathcal{E}^{1}, \mathcal{I}^{1}, d^{1}\right)$ defined on a maximal contact hypersurface $N^{1}$ of $Z$ (in some neighbourhood of $a$ ); see [3, §5.9]. Here $\mathcal{E}^{1}=\mathcal{E}^{1}(a)=E(a) \backslash E^{1}(a)$. The boundary is the marked ideal determined by the old components $E^{1}(a)$ of the exceptional divisor $E(a)$. The maximal contact hypersurface is transformed from the year of birth of $\operatorname{inv}_{1 / 2}(a)$, so it is transverse to the set of new components $\mathcal{E}^{1}(a)$ of $E(a)$. It need not be transverse to $E^{1}(a)$; the boundary is therefore added to the coefficient ideal to ensure the centre of blowing up will lie inside the old components.

Iterating the process, we will get a coefficient ideal plus boundary $\underline{\mathcal{I}}^{k}=$ $\left(Z, N^{k}, \mathcal{E}^{k}, \mathcal{I}^{k}, d^{k}\right)$ defined on a maximal contact subspace $N^{k}$ of codimension $k$ in $Z$, in a neighbourhood of $a$. The boundary here is given by $E^{k}(a)$, and $\mathcal{E}^{k}=$ $\mathcal{E}^{k}(a):=\mathcal{E}^{k-1}(a) \backslash E^{k}(a)=E(a) \backslash E^{1}(a) \cup \cdots \cup E^{k}(a)$.

We again write $\mathcal{I}^{k}$ as the product $\mathcal{M}\left(\underline{\mathcal{I}}^{k}\right) \cdot \mathcal{R}\left(\underline{\mathcal{I}}^{k}\right)$ of its monomial and residual parts. $\mathcal{M}\left(\underline{\mathcal{I}}^{k}\right)$ is the monomial part with respect to $\mathcal{E}^{k}$; i.e., it is the product of the ideals $\mathcal{I}_{H}, H \in \mathcal{E}^{k}(a)$, each to the power $\operatorname{ord}_{H, a} \mathcal{I}^{k}$, where ord ${ }_{H, a}$ denotes the order along $H$ at $a$. We set $\nu_{k+1}(a):=\operatorname{ord}_{a} \mathcal{R}\left(\underline{\mathcal{I}}^{k}\right) / d^{k}$ and $\mu_{H, k+1}(a):=\operatorname{ord}_{H, a} \mathcal{I}^{k} / d^{k}$, $H \in \mathcal{E}^{k}(a)$; both are invariants of the equivalence class of $\underline{\mathcal{I}}^{k}$ and $\operatorname{dim} N^{k}$ (see [3, Def. 5.10]). The iterative construction terminates when $\nu_{k+1}(a)=0$ or $\infty$.

In Section 3 (see Lemma 3.1), we will show that, at an snc point in any year of the resolution history, the invariant takes a special form:

Definition 2.1. Given $p \in \mathbb{N}, d \geq 1$ and $s=\left(s_{1}, \ldots, s_{d}\right)$ with each $s_{k} \in \mathbb{N}$, set

$$
\operatorname{inv}_{p, s}:=\left(p, s_{1}, 1, s_{2}, \ldots, 1, s_{d}, 1,0, \ldots, 1,0, \infty\right),
$$

where the total number of pairs (before $\infty$ ) is

$$
r:=p+|s|, \quad|s|:=\sum_{k=1}^{d} s_{k} .
$$

In particular, if $E=\emptyset$, then, at an snc point in year zero, inv $=\operatorname{inv}_{p, 0}=$ $(p, 0,1,0, \ldots, 1,0, \infty)$ with $p$ pairs. The algorithm of [3, Thm. 3.4] depends on a characterization of points with inv $=\operatorname{inv}_{p, 0}$.

The following example distinguishes the algorithm of Theorem 1.4 (which we will call Algorithm C) from those of the weaker result [3, Thm. 3.4] (Algorithm B) and the standard desingularization algorithm [1, 2] (Algorithm A). See also Example 5.2. The table below provides the computations of marked ideals needed to find the invariant and the centre $C$ of the blowing-up at the origins of the charts corresponding to the coordinate substitutions indicated. The calculations at a given point provide the next centre of blowing up over a neighbourhood of that point; globally, the maximum locus of the invariant will be blown up first.

In each subtable, the passage from $\underline{\mathcal{J}}^{k}$ to $\underline{\mathcal{I}}^{k+1}$ is given by taking the coefficient ideal plus boundary, on the maximal contact subspace of codimension $k+1$.

Example 2.2. Consider the hypersurface $X \hookrightarrow \mathbb{A}^{3}$ given by $\left(z^{3}+x y=0\right)$, together with the empty divisor $E=\emptyset$. The following table computes blowings-up given by the standard resolution algorithm A. 


\begin{tabular}{c|c|c|c|c}
\hline codim & marked ideal & companion ideal & maximal & boundary \\
$i$ & $\underline{\mathcal{I}}^{i}$ & $\underline{\mathcal{J}}^{i}=\underline{\mathcal{G}}\left(\underline{\mathcal{I}}^{i}\right)$ & contact & $E^{i+1}$ \\
\hline
\end{tabular}

Year zero.

\begin{tabular}{c|c|c|c|c}
\hline 0 & $\left(z^{3}+x y, 1\right)$ & $\left(z^{3}+x y, 2\right)$ & $(x=0)$ & \\
\hline 1 & $\left(\left(z^{3}, y^{2}\right), 2\right)$ & $\left(\left(z^{3}, y^{2}\right), 2\right)$ & $(x=y=0)$ & \\
\hline 2 & $\left(z^{3}, 2\right)$ & $\left(z^{3}, 3\right)$ & $(x=y=z=0)$ & \\
\hline 3 & $(0)$ & & & \\
$\operatorname{inv}(0)(2,0,1,0,3 / 2,0, \infty), C_{0}=\{0\}$
\end{tabular}

Year one. Coordinate chart $(x z, y z, z)$

\begin{tabular}{c|c|c|c|c}
\hline 0 & $(z(z+x y), 1)$ & $(z+x y, 1)$ & $(z+x y=0)$ & $(z=0)$ \\
\hline 1 & $(x y, 1)$ & $(x y, 2)$ & $(x=z=0)$ & \\
\hline 2 & $(y, 1)$ & $(y, 1)$ & $(x=y=z=0)$ & \\
\hline 3 & $(0)$ & & & \\
\hline
\end{tabular}

$\operatorname{inv}(0)=(1,1,2,0,1,0, \infty), C_{1}=\{0\}$

Year two. Coordinate chart $(x, x y, x z)$

\begin{tabular}{c|c|c|c|c}
\hline 0 & $(x z(z+x y), 1)$ & $(z+x y, 1)$ & $(z+x y=0)$ & $(z=0)$ \\
\hline 1 & $(x y, 1)$ & $(y, 1)$ & $(y=z=0)$ & $(x=0)$ \\
\hline 2 & $(x, 1)$ & $(x, 1)$ & $(x=y=z=0)$ & \\
\hline 3 & $(0)$ & & & \\
\hline
\end{tabular}

$\operatorname{inv}(0)=(1,1,1,1,1,0, \infty), C_{2}=\{0\}$

Year three. Coordinate chart $(x, x y, x z)$

\begin{tabular}{c|c|c|c|c}
\hline 0 & $\left(x^{2} z(z+x y), 1\right)$ & $(z+x y, 1)$ & $(z+x y=0)$ & $(z=0)$ \\
\hline 1 & $(x y, 1)$ & $(y, 1)$ & $(y=z=0)$ & \\
\hline 2 & $(0)$ & & & \\
\hline
\end{tabular}

Note that none of the invariants computed are special values of the form $\operatorname{inv}_{p, 0}$. Therefore, Algorithm B coincides with A at each of the steps shown. In year three, the centre $C_{3}$ includes snc points (though not snc points that were present in year zero), so that both Algorithms A and B blow up snc points in year three.

On the other hand, in year two, $\operatorname{inv}(0)$ is a special value $\operatorname{inv}_{p, s}$, where $p=1$ and $s=(1,1)$. Algorithm $\mathrm{C}$ nevertheless blows up $C_{2}=\{0\}$, as do Algorithms $\mathrm{A}$ 
and $\mathrm{B}$, because $C_{2}$ has no snc points (see step (1) in Section 5). In year three, $\operatorname{inv}(0)$ is again a special value $\operatorname{inv}_{p, s}$, where now $p=1$ and $s=(1)$. Algorithm $\mathrm{C}$ then continues with a cleaning blowing-up, with centre $(x=z=0)$, after which we have simple normal crossings over the year-three chart.

\section{Characterization of Simple normal CRossings}

Consider $X \hookrightarrow Z$ and $E$ as in Definition 1.1 and the sequence of inv-admissible blowings-up given by the standard desingularization algorithm. Throughout the remainder of the paper, we will assume that $X$ and $E$ have no components in common (for technical convenience only - Theorem 1.4 as stated follows easily from this case). Lemma 3.1 below asserts that inv takes a special form inv $\operatorname{los}_{p, s}$ at an snc point in any year of the resolution history. The converse is not true. For example, if $X=\left(x_{1}^{n}+\cdots+x_{n}^{n}=0\right)$ and $E=\emptyset$, then $\operatorname{inv}(0)=(n, 0,1,0,1, \ldots, 0, \infty)=\operatorname{inv}_{n, 0}$ (in year zero). However, if we make the additional assumption that $X$ (or its strict transform in a given year) has $p$ irreducible components at a point $a$ with $\operatorname{inv}(a)=\operatorname{inv}_{p, s}$, then we can write local normal forms for the components of $X$ and $E$ at $a$ (Lemma 3.3) and we can characterize snc using inv and the additional invariants $\mu_{H, k}(a)$ defined in Section 2 or [3, Def. 5.10] (Theorem 3.4 below). We begin by stating all three results. The proofs of Lemmas 3.1 and 3.3 follow parallel arguments, so we give them together.

Lemma 3.1. Suppose $(X, E)=\left(X_{m}, E_{m}\right)$ is snc at a point a, in some year $m$ of the resolution history (1.1). Then $\operatorname{inv}(a)$ is of the form $\operatorname{inv}_{p, s}$ where $r=p+|s| \leq n$ (see Definition 2.1). Moreover, the invariants $\mu_{H, i+1}(a)=0$, for all $i \geq 1$ and $H \in E(a)$.

Definition 3.2. Let $\Sigma_{p}=\Sigma_{p}(X)$ denote the set of points lying in $p$ irreducible components of $X$.

Lemma 3.3. Let $a \in X=X_{m}$. Assume that $a \in \Sigma_{p}(X)$ and $\operatorname{inv}(a)=\operatorname{inv}_{p, s}, s=$ $\left(s_{1}, \ldots, s_{d}\right)$. Let $f_{k}, k=1, \ldots, p$, denote generators of the ideals of the components of $X$ at $a$, and let $u_{i}^{j}, j=1, \ldots, s_{i}$, denote generators of the ideals of the elements of $E^{i}(a), i=1, \ldots, d$.

Set $r:=p+|s|$. Then there is a bijection $\{1, \ldots, r\} \rightarrow\left\{f_{k}, u_{i}^{j}\right\}$, which we denote $l \mapsto g_{l}$, and a regular system of coordinates $\left(x_{1}, \ldots, x_{n}\right)$ at $a(n \geq r)$, such that

$$
\begin{aligned}
& g_{1}=f_{1}=x_{1}, \\
& g_{l}=\xi_{l}+x_{l} \prod_{i=1}^{l-1} m_{i+1}, \quad l=2, \ldots, r,
\end{aligned}
$$

where each $\xi_{l}$ is in the ideal generated by $\left(x_{1}, \ldots, x_{l-1}\right)$ and each $m_{i+1}$ is a monomial in generators of the ideals of the elements $H$ of $\mathcal{E}^{i}(a)=E(a) \backslash E^{1}(a) \cup \cdots \cup E^{i}(a)$, each raised to the power $\mu_{H, i+1}(a)$.

Theorem 3.4 (Characterization of snc). Let $a \in X=X_{m}$. Then $(X, E)$ is snc at $a$ if and only if:

(1) $a \in \Sigma_{p}(X)$, for some $p \geq 1$,

(2) $\operatorname{inv}(a)=\operatorname{inv}_{p, s}$, for some $s=\left(s_{1}, \ldots, s_{d}\right)$,

(3) $\mu_{H, i+1}(a)=0$, for all $i \geq 1$ and all $H \in \mathcal{E}^{i}(a)$.

Proof. Immediate from Lemmas 3.1 and 3.3 . 
We will need the following simple lemma (see [3, §5.4]).

Lemma 3.5. Let $a \in N \subset Z$, where $N$ is a smooth subvariety of a neighbourhood of $a$, and let $f_{1}, \ldots, f_{p}$ denote regular functions of order one on $N$. Then the marked ideals $\underline{\mathcal{I}}_{1}=\left(Z, N, \emptyset,\left(f_{1} \cdots f_{p}\right), p\right)$ and $\underline{\mathcal{I}}_{2}=\left(Z, N, \emptyset,\left(f_{1}, \ldots, f_{p}\right), 1\right)$ are equivalent. (In particular, any sequence of blowings-up which is admissible for one is also admissible for the other.)

Proof of Lemmas 3.1 and 3.3. Consider $(X, E)=\left(X_{m}, E_{m}\right)$ in some year $m$ of the resolution history (1.1), and consider $a \in \Sigma_{p}(X)$. Let $f_{k}, k=1, \ldots, p$, denote generators of the ideals of the components of $X$ at $a$. The computation of $\operatorname{inv}(a)$ begins with the marked ideal $\underline{\mathcal{I}}^{0}=\left(Z, Z, E(a), \mathcal{I}^{0}, 1\right)$, where $\mathcal{I}^{0}=\left(f_{1} \cdots f_{p}\right)$ times a monomial in the components of $E(a)$. Clearly, $\operatorname{inv}_{1 / 2}(a)=p$ if and only if $\operatorname{ord}_{a} f_{k}=$ 1 for all $k$. Assume the latter. Then $\mathcal{R}\left(\underline{\mathcal{I}}^{0}\right)=\left(f_{1} \cdots f_{p}\right)$ and the companion ideal $\underline{\mathcal{J}}^{0}=\left(E(a),\left(f_{1} \cdots f_{p}\right), p\right)$. Any $\left(f_{k}=0\right)$, say $\left(f_{1}=0\right)$, defines a hypersurface of maximal contact $N^{1}$ for $\left(\mathcal{E}(a),\left(f_{1} \cdots f_{p}\right), p\right)$ or, equivalently, $\left(E(a),\left(f_{1}, \ldots, f_{p}\right), 1\right)$. (This is a consequence of Lemma 3.5 and the fact that, if $m^{\prime}$ is the year of birth of $\operatorname{inv}_{1 / 2}(a)$, then $X_{m^{\prime}}$ has $p$ components of order 1 at $a_{m^{\prime}}$.) Let $x_{1}=g_{1}:=f_{1}$.

Let $u_{1}^{j}, j=1, \ldots, s_{1}$, denote generators of the ideals of the elements of $E^{1}(a)$. Then the coefficient ideal plus boundary is

$$
\underline{\mathcal{I}}^{1}=\left(Z, N^{1}, \mathcal{E}^{1}(a),\left.\left(f_{2}, \ldots, f_{p}, u_{1}^{1}, \ldots, u_{1}^{s_{1}}\right)\right|_{N^{1}}, 1\right),
$$

where $\mathcal{E}^{1}(a)=E(a) \backslash E^{1}(a)$. (Note, in particular, that the associated multiplicity is 1.)

We factor $\underline{\mathcal{I}}^{1}$ as the product $\mathcal{M}\left(\underline{\mathcal{I}}^{1}\right) \cdot \mathcal{R}\left(\underline{\mathcal{I}}^{1}\right)$ of its monomial and residual parts; in particular, $\mathcal{M}\left(\underline{\mathcal{I}}^{1}\right)$ is generated by a monomial $m_{2}$ in the components of $\mathcal{E}^{1}(a)$.

First suppose that $(X, E)$ is snc at $a$. Then the generators of $\mathcal{I}^{1}$ in (3.2) are part of a regular coordinate system. It follows that $\mathcal{M}\left(\underline{I}^{1}\right)=1$ (since none of these generators define elements of $\left.\mathcal{E}^{1}(a)\right)$; i.e., all $\mu_{H, 2}(a)=0$. Since $\underline{\mathcal{I}}^{1}$ has maximal order, $\operatorname{inv}_{3 / 2}(a)=\left(p, s_{1}, 1\right)$, and the next companion ideal $\underline{\mathcal{J}}^{1}=\underline{\mathcal{I}}^{1}$.

We can then continue as before, choosing the $f_{k}$ and the $u_{i}^{j}$ successively as hypersurfaces of maximal contact to pass to the coefficient ideal plus boundary $\underline{\mathcal{I}}^{\ell}$, $\ell=2, \ldots$ At each step, $\mathcal{M}\left(\underline{\mathcal{I}}^{\ell}\right)=1$ (in particular, $\mu_{H, \ell+1}(a)=0$ for every $H$ ), and $\underline{\mathcal{I}}^{\ell}$ is of maximal order, $=1$. Therefore, $\nu_{\ell+1}=1$ and $\underline{\mathcal{I}}^{\ell}$ equals the following companion ideal $\underline{\mathcal{J}}^{\ell}$. Once all $f_{k}$ and $u_{i}^{j}$ have been used as hypersurfaces of maximal contact, we get coefficient ideal $=0$. Therefore, $\operatorname{inv}(a)$ has last entry $=\infty$ and $r$ pairs before $\infty$. This completes the proof of Lemma 3.1.

Now consider the converse direction for Lemma 3.3. If $\operatorname{inv}_{3 / 2}(a)=\left(p, s_{1}, 1\right)$, then there exists $g_{2} \in\left\{f_{2}, \ldots, f_{p}, u_{1}^{1}, \ldots, u_{1}^{s_{1}}\right\}$ such that $x_{2}:=\left.m_{2}^{-1} \cdot g_{2}\right|_{N^{1}} \in \mathcal{R}\left(\underline{\mathcal{I}}^{1}\right)$ has order 1 at $a$, and the next companion ideal $\underline{\mathcal{J}}^{1}=\left(Z, N^{1}, \mathcal{E}^{1}(a), \mathcal{R}\left(\underline{\mathcal{I}}^{1}\right), 1\right)$. We can take $N^{2}:=\left(x_{2}=0\right) \subset N^{1}$ as the next maximal contact subspace, and write $g_{2}=\xi_{2}+x_{2} m_{2}$, where $\xi_{2} \in\left(x_{1}\right)$. Then the coefficient ideal plus boundary is

$$
\underline{\mathcal{I}}^{2}=\left(Z, N^{2}, \mathcal{E}^{2}(a)=\mathcal{E}^{1}(a) \backslash E^{2}(a),\left.\left(\mathcal{R}\left(\underline{\mathcal{I}}^{1}\right)+\left(u_{2}^{1}, \ldots, u_{2}^{s_{2}}\right)\right)\right|_{N^{2}}, 1\right) .
$$

We can again repeat the argument. At each step $\ell \geq 2$, the ideal $\mathcal{I}^{\ell}$ factors as $\mathcal{M}\left(\underline{\mathcal{I}}^{\ell}\right) \cdot \mathcal{R}\left(\underline{\mathcal{I}}^{\ell}\right)$ where $\mathcal{M}\left(\underline{\mathcal{I}}^{\ell}\right)$ is generated by a monomial $m_{\ell+1}$ in the components of $\mathcal{E}^{\ell}(a)=E(a) \backslash E^{1}(a) \cup \cdots \cup E^{\ell}(a)$. If the truncated invariant $\operatorname{inv}_{\ell+1 / 2}(a)=\left(\operatorname{inv}_{p, s}\right)_{\ell+1 / 2}$ (in particular, $\left.\nu_{\ell+1}(a)=1\right)$, then $\mathcal{R}\left(\underline{\mathcal{I}}^{\ell}\right)$ has maximal order 1 . Therefore, the next companion ideal $\underline{\mathcal{J}}^{\ell}=\left(Z, N^{\ell}, \mathcal{E}^{\ell}(a), \mathcal{R}\left(\underline{\mathcal{I}}^{\ell}\right), 1\right)$, where 
$N^{\ell}=\left(x_{1}=\ldots=x_{\ell}=0\right)$. Therefore, there is $g_{\ell+1} \in\left\{f_{2}, \ldots, f_{p}, u_{1}^{1}, u_{1}^{2}, \ldots, u_{\ell}^{s_{\ell}}\right\}$ such that $x_{\ell+1}:=\left.\prod_{i=1}^{\ell} m_{i+1}^{-1} \cdot g_{\ell+1}\right|_{N^{\ell}} \in \mathcal{R}\left(\underline{\mathcal{I}}^{\ell}\right)$ and has order 1 . We can take $\left(x_{\ell+1}=0\right)$ as the next hypersurface of maximal contact $N^{\ell+1} \subset N^{\ell}$ and write $g_{\ell+1}=\xi_{\ell+1}+x_{\ell+1} \prod_{i=1}^{\ell} m_{i+1}$, for some $\xi_{\ell+1} \in\left(x_{1}, \ldots, x_{\ell}\right)$.

If $\operatorname{inv}(a)=\operatorname{inv}_{p, s}$, then the process ends after $r=p+|s|$ steps (i.e., $r$ successive choices of maximal contact) with $\underline{\mathcal{I}}^{r}=0$.

Remark 3.6. In the proof above, we have noted that, if $a \in \Sigma_{p}$ and the truncated invariant $\operatorname{inv}_{k+1 / 2}(a)=\left(\operatorname{inv}_{p, s}\right)_{k+1 / 2}$, where $0 \leq k<r=p+|s|$, then, for every $\ell \leq k+1$, the coefficient ideal plus boundary $\underline{\mathcal{I}}^{\ell}$ (or an equivalent marked ideal) has associated multiplicity $=1$.

\section{Cleaning}

According to Theorem 3.4, if $a \in \Sigma_{p}$ and $\operatorname{inv}(a)=\operatorname{inv}_{p, s}$, then $(X, E)$ is snc at $a$ if and only if the invariants $\mu_{H, k+1}(a)=0$, for every $k \geq 1$. In this section we study the cleaning blowings-up necessary to get the latter condition.

The centres of cleaning blowings-up are not necessarily inv-admissible. In the general cleaning algorithm of [3, Sect.2], therefore, inv is not defined in a natural way over a cleaning sequence, so that, after cleaning, we assume we are in year zero for the definition of inv. Over the particular cleaning sequences needed here, however, we can define a modified inv which remains semicontinuous and infinitesimally semicontinuous, and show that maximal contact subspaces exist in every codimension involved; this is a consequence of Lemma 3.3 and Remark 3.6 (see Remarks 4.3).

The cleaning procedure provides one of the two main steps that are iterated to give the algorithm for Theorem 1.4 (Algorithm C). The algorithm is presented in Section 5. Example 5.2 illustrates the cleaning procedure and, in particular, how the modified inv is computed after a cleaning blowing-up.

Consider a point $a$ in the locus $S:=\left(\operatorname{inv}_{k}=\left(\operatorname{inv}_{p, s}\right)_{k}\right)$, where $k \geq 1$ (in any year $m$ of the resolution history (1.1)). In some neighbourhood of $a, S$ is the cosupport of a marked ideal (a coefficient ideal plus boundary) $\underline{\mathcal{I}}^{k}=\left(\mathcal{I}^{k}, d^{k}\right)=$ $\left(Z, N^{k}, \mathcal{E}^{k}(a), \mathcal{I}^{k}, d^{k}\right)$, where $N^{k}$ is a maximal contact subspace of codimension $k$ and $d^{k}=1$ (see Remark 3.6). Recall that $\mathcal{E}^{k}(a)=E(a) \backslash E^{1}(a) \cup \cdots \cup E^{k}(a)$. $\mathcal{E}^{k}(a)$ is the block of new components of the exceptional divisor (necessarily transverse to $N^{k}$ ) and the old components in the block $E^{k}(a)$ define the boundary.

The ideal $\mathcal{I}^{k}=\mathcal{M}\left(\underline{\mathcal{I}}^{k}\right) \cdot \mathcal{R}\left(\underline{\mathcal{I}}^{k}\right)$ (the product of its monomial and residual parts). The monomial part $\mathcal{M}\left(\underline{\mathcal{I}}^{k}\right)$ is the product of the ideals $\left.\mathcal{I}_{H}\right|_{N^{k}}$ (where $H \in \mathcal{E}^{k}(a)$ ), each to the power $\mu_{H, k+1}(a)$ (since $d^{k}=1$ ).

Let $\underline{\mathcal{M}}\left(\underline{\mathcal{I}}^{k}\right)$ denote the monomial marked ideal $\left(\mathcal{M}\left(\underline{\mathcal{I}}^{k}\right), d^{k}\right)=\left(\mathcal{M}\left(\underline{\mathcal{I}}^{k}\right), 1\right)$. Then $\operatorname{cosupp} \underline{\mathcal{M}}\left(\underline{\mathcal{I}}^{k}\right) \subset \operatorname{cosupp} \underline{\mathcal{I}}^{k}$ and any admissible sequence of blowings-up of $\underline{\mathcal{M}}\left(\underline{\mathcal{I}}^{k}\right)$ is admissible for $\underline{\mathcal{I}}^{k}$. Cleaning is provided by desingularization of the monomial marked ideal $\underline{\mathcal{M}}\left(\underline{\mathcal{I}}^{k}\right)$ [2, Sect.5, Step II, Case A], [3, Sect.2]; see Definition 4.2 below.

Remark 4.1. First note that, if $X$ has constant order on an irreducible subvariety $C$, then, for any $p$, either $C \subset \Sigma_{p}(X)$ or $C \cap \Sigma_{p}(X)=\emptyset$ (by semicontinuity of order). 
Definition 4.2. Cleaning of the locus $S=\left(\operatorname{inv}_{k}=\left(\operatorname{inv}_{p, s}\right)_{k}\right)$ means the sequence of blowings-up obtained from desingularization of the monomial marked ideal $\underline{\mathcal{M}}\left(\underline{\mathcal{I}}^{k}\right)$ (in a neighbourhood of any point of $S$ ) by using, at each step, only the components of the centres of blowing ups which lie in $\Sigma_{p}$.

The centres of the cleaning blowings-up are invariantly defined closed subspaces of $\left(\operatorname{inv}_{k} \geq\left(\operatorname{inv}_{p, s}\right)_{k}\right)$.

Remarks 4.3. The blowings-up $\sigma$ involved in desingularization of $\underline{\mathcal{M}}\left(\underline{\mathcal{I}}^{k}\right)$ are admissible: Let $C$ denote the centre of $\sigma$. Then $C$ is snc with respect to $E$ because, in the notation above, $C$ lies inside every element of $E^{1}(a) \cup \cdots \cup E^{k}(a)$ and $C$ is snc with respect to $\mathcal{E}^{k}(a)$. Since $C \subset S$, it follows that $\sigma$ is $\operatorname{inv}_{k}$-admissible. By Lemma 3.1. $C$ contains no snc points (since some $\mu_{H, k+1}(a) \neq 0$, for all $a \in C$ ).

Since $d_{k}=1, C$ is of the form $N^{k} \cap H$, for a single $H \in \mathcal{E}^{k}(a)$; i.e., $C$ is of codimension 1 in $N^{k}$. Therefore, $\sigma$ induces an isomorphism $\left(N^{k}\right)^{\prime} \rightarrow N^{k}$, where $\left(N^{k}\right)^{\prime}$ denotes the strict transform of $N^{k}$.

Lemma 4.4. Assume that inv $\leq \operatorname{inv}_{p, s}$ on $X=X_{m}$, in some year $m$ of the desingularization history. Consider the cleaning sequence for $S=\left(\operatorname{inv}_{k}=\left(\operatorname{inv}_{p, s}\right)_{k}\right)$ (Definition 4.2). Then, over the cleaning sequence, we can define maximal contact subspaces of every codimension involved, as well as (a modification of) inv which remains both semicontinuous and infinitesimally semicontinuous.

Proof. Consider the first blowing-up $\sigma$ in the cleaning sequence, at a point $a$ as above. Let $C$ denote the centre of $\sigma$.

Since $d^{k}=1, C \subset N^{k}$ is given by $H \cap N^{k}$, for some $H \in \mathcal{E}^{k}(a)$. Let $\left(\underline{\mathcal{I}}^{k}\right)^{\prime}=$ $\left(Z^{\prime},\left(N^{k}\right)^{\prime},\left(\mathcal{E}^{k}\right)^{\prime},\left(\mathcal{I}^{k}\right)^{\prime}, 1\right)$ denote the transform of $\underline{\mathcal{I}}^{k}$ by $\sigma$ (in particular, $Z=Z_{m+1}$; i.e., this is year $m+1$ ). Then $\left.\sigma\right|_{\left(N^{k}\right)^{\prime}}:\left(N^{k}\right)^{\prime} \rightarrow N^{k}$ is an isomorphism (which we consider to be the identity), so that $\sigma^{*}\left(\mathcal{I}^{k}\right)=\mathcal{I}^{k}$. Therefore, $\left(\mathcal{I}^{k}\right)^{\prime}=\mathcal{I}_{H}^{-1} \sigma^{*}\left(\mathcal{I}^{k}\right)=$ $\mathcal{I}_{H}^{-1} \mathcal{I}^{k}$ and

$$
\mathcal{R}\left(\left(\mathcal{I}^{k}\right)^{\prime}\right)=\mathcal{R}\left(\mathcal{I}^{k}\right), \quad \mathcal{M}\left(\left(\mathcal{I}^{k}\right)^{\prime}\right)=\mathcal{I}_{H}^{-1} \mathcal{M}\left(\mathcal{I}^{k}\right) .
$$

Over the complement of $C, \sigma$ is an isomorphism and inv will be unchanged. Consider $a^{\prime} \in \sigma^{-1}(a)$. If $a^{\prime} \notin\left(N_{k}\right)^{\prime}$, then $\operatorname{inv}_{k}\left(a^{\prime}\right)<\operatorname{inv}_{k}(a)$, by the definition of maximal contact (since $\sigma$ is $\operatorname{inv}_{k^{-}}$-admissible). For any $a^{\prime} \in \sigma^{-1}(a)$ such that $\operatorname{inv}_{k}\left(a^{\prime}\right)<\operatorname{inv}_{k}(a), \operatorname{inv}_{k}\left(a^{\prime}\right)$ can be extended to $\operatorname{inv}\left(a^{\prime}\right)$ as in the usual desingularization algorithm. (This is "year zero" for $\operatorname{inv}_{k}\left(a^{\prime}\right)$.)

On the other hand, suppose $a \in\left(N_{k}\right)^{\prime}$ and $\operatorname{inv}_{k}\left(a^{\prime}\right)=\operatorname{inv}_{k}(a)$. Following the standard desingularization algorithm, we set $\nu_{k+1}\left(a^{\prime}\right)=\operatorname{ord}_{a^{\prime}} \mathcal{R}\left(\left(\mathcal{I}^{k}\right)^{\prime}\right)=\nu_{k+1}(a)$. Then $\operatorname{inv}_{k+1 / 2}=\left(\operatorname{inv}_{k}, \nu_{k+1}\right)$ is semicontinuous on $Z^{\prime}=Z_{m+1}$ and infinitesimally semicontinuous through to year $m+1$. The exponents $\mu_{H, k+1}\left(a^{\prime}\right)$ in $\mathcal{M}\left(\left(\mathcal{I}^{k}\right)^{\prime}\right)$ and $\nu_{k+1}\left(a^{\prime}\right)$ are invariants of the equivalence class of $\left(\underline{\mathcal{I}}^{k}\right)^{\prime}$ (cf. [3], Def. $5.10 \mathrm{ff}$.]).

Recall that the companion ideal $\underline{\mathcal{J}}^{k}$ of $\underline{\mathcal{I}}^{k}$ is $\left(\mathcal{R}\left(\mathcal{I}^{k}\right), 1\right)$ (as in the proof of Lemma 3.3). The equivalence class of the companion ideal $\underline{\mathcal{J}}_{m+1}^{k}=\left(\underline{\mathcal{J}}^{k}\right)^{\prime}$ of $\underline{\mathcal{I}}_{m+1}^{k}=\left(\underline{\mathcal{I}}^{k}\right)^{\prime}$ depends only on the equivalence class of $\left(\underline{\mathcal{I}}^{k}\right)^{\prime}$ (cf. [2, Cor. 5.3]).

If $\nu_{k+1}(a)>0$, then $\mathcal{J}^{k}$ admits a maximal contact hypersurface $N^{k+1} \subset N^{k}$ at $a$ (relative to $\mathcal{E}^{k+1}(a)$; in particular, $N^{k+1}$ is transverse to $\mathcal{E}^{k+1}$ at $a$ ). If we were to follow the standard desingularization algorithm, then we would take $s_{k+1}\left(a^{\prime}\right):=$ $\# E^{k+1}\left(a^{\prime}\right)$, where $E^{k+1}\left(a^{\prime}\right)$ is the set of strict transforms at $a^{\prime}$ of elements of $E^{k+1}(a)$, and we would include the exceptional divisor $\widetilde{H}=\sigma^{-1}(C)$ of $\sigma$ in $\mathcal{E}^{k+1}\left(a^{\prime}\right)$. There are two cases to be considered. 
(1) $H \notin E^{k+1}(a)$. Then $s_{k+1}\left(a^{\prime}\right)=s_{k+1}(a)$. Since $H \in \mathcal{E}^{k+1}(a), H$ is transverse to $N^{k+1}$. Therefore, $\widetilde{H}$ is transverse to $\left(N^{k+1}\right)^{\prime}$, and $\left(N^{k+1}\right)^{\prime}$ is a valid maximal contact subspace of codimension $k+1$ at $a^{\prime}$.

(2) $H \in E^{k+1}(a)$. Then $H$ is not necessarily transverse to $N^{k+1}$ and $\widetilde{H}:=$ $\sigma^{-1}(C)$ is not necessarily transverse to $\left(N^{k+1}\right)^{\prime}$, so that $\left(N^{k+1}\right)^{\prime}$ is not valid as a maximal contact subspace of codimension $k+1$ at $a^{\prime}$. Instead, we modify the definition of $\operatorname{inv}_{k+1}$ and the algorithm in this case by continuing to count $\widetilde{H}$ as an element of $E^{k+1}\left(a^{\prime}\right)$ (so we are viewing $\left(N^{k}\right)^{\prime} \cap \widetilde{H}$ simply as a relabelling of $N^{k} \cap H$; i.e., $H$ is moved away from $N^{k}$ and replaced by $\left.\widetilde{H}\right)$. Thus $s_{k+1}\left(a^{\prime}\right)=s_{k+1}(a)$ and $\mathcal{E}^{k+1}\left(a^{\prime}\right)$ is still transverse to $\left(N^{k+1}\right)^{\prime}$.

In both cases, it follows that the equivalence class of the coefficient ideal plus boundary $\underline{\mathcal{I}}_{m+1}^{k+1}$ associated to $\underline{\mathcal{J}}_{m+1}^{k}=\left(\underline{\mathcal{J}}^{k}\right)^{\prime}$ depends only on the equivalence class of $\underline{\mathcal{J}}_{m+1}^{k}$ and $E^{k+1}\left(a^{\prime}\right)$ [2, Sect.5, Step I, Case B]. Therefore, $\operatorname{inv}_{k+1}=$ $\left(\operatorname{inv}_{k}, \nu_{k+1}, s_{k+1}\right)$ extends as in the standard desingularization algorithm to inv on $Z_{m+1}$. Clearly, if $\operatorname{inv}_{k}\left(a^{\prime}\right)=\operatorname{inv}_{k}(a)$, where $a \in C$ as above, then $\operatorname{inv}\left(a^{\prime}\right)=\operatorname{inv}(a)$.

The argument above can be iterated over the full sequence of cleaning blowingsup. The order of the blowings-up in the cleaning sequence (i.e., in desingularization of the corresponding monomial marked ideal) is determined by the ordering of $\mathcal{E}^{k}$ given by the year of birth of each of its elements; see [2, Sect. 5, Step II, Case A].

Remark 4.5. After cleaning the loci $\left(\operatorname{inv}_{k}=\left(\operatorname{inv}_{p, s}\right)_{k}\right)$, for all $k,\left(\operatorname{inv}=\operatorname{inv}_{p, s}\right)$ becomes snc. We will then continue to blow up with closed centres that lie in the complement of $\{\mathrm{snc}\}$ (Section 5). The purpose of defining inv over the cleaning sequences is to ensure that, in the complement of $\{\mathrm{snc}\}$, we will only have to consider values $\operatorname{inv}_{p^{\prime}, s^{\prime}}<\operatorname{inv}_{p, s}$ in order to resolve all but $\{\mathrm{snc}\}$ after finitely many steps. If, after cleaning (inv $=\operatorname{inv}_{p, s}$ ), we were to apply the resolution algorithm in the complement of $\{\mathrm{snc}\}$, beginning as if in year zero, we might introduce points where inv $=\operatorname{inv}_{p^{\prime}, s^{\prime}}>\operatorname{inv}_{p, s}$.

\section{Algorithm for the main theorem}

In this section we prove Theorem[1.4 Let $\mathcal{S}$ denote $\left\{\operatorname{inv}_{p, s}\right\}$ where $s=\left(s_{1}, \ldots, s_{d}\right)$ and $p, d,|s| \leq n:=\operatorname{dim} Z$. Then $\mathcal{S}$ is finite and totally ordered. Consider the following two steps:

(1) Let $\operatorname{inv}_{p, s}$ be the maximum element of $\mathcal{S}$. Follow the desingularization algorithm of [1, 2] to decrease inv until inv is everywhere $\leq \operatorname{inv}_{p, s}$. Then blow up any component of the locus $\left(i n v=\operatorname{inv}_{p, s}\right.$ ) that contains only non-snc points. The result is that $(X, E)$ is generically snc on every component of the locus (inv $\left.=\operatorname{inv}_{p, s}\right)$. By Remark 4.1] (inv $\left.=\operatorname{inv}_{p, s}\right) \subset \Sigma_{p}(X)$.

(2) Clean the locus $\left(\operatorname{inv}_{k}=\left(\operatorname{inv}_{p, s}\right)_{k}\right)$, successively for each $k=r-1, r-2, \ldots, 1$, where $r=p+|s|$, using Lemma 4.4. Then all $\mu_{H, k}=0,2 \leq k \leq r$, so, by Theorem [3.4 all points of $\left(\mathrm{inv}=\operatorname{inv}_{p, s}\right)$ are snc. Therefore, $(X, E)$ is snc on $Y_{p, s}:=\left(\operatorname{inv} \geq \operatorname{inv}_{p, s}\right)$, and hence lies in a neighbourhood of $Y_{p, s}$.

We can therefore repeat the above two steps on $X_{p, s}:=X \backslash Y_{p, s}$, replacing $\mathcal{S}$ by $\mathcal{S} \backslash\left\{\operatorname{inv}_{p, s}\right\}$. The centres of all blowings-up involved are closed in $X$ since they are closed in $X_{p, s}$ and contain no snc points (according to Theorem 3.4 and Remarks 4.3).

Since $\mathcal{S}$ is finite, the process terminates after finitely many iterations, so that $(X, E)$ becomes everywhere snc. 
The desingularization algorithm [2] is functorial with respect to étale or smooth morphisms. The snc condition is preserved by étale or smooth morphisms which preserve the number of irreducible components at every point. The functoriality statement in Theorem 1.4 is an immediate consequence (cf. [4, Sect.9]). This completes the proof.

Remark 5.1. The algorithm above admits certain variations; e.g., (1) can be changed so that, when inv $\leq \operatorname{inv}_{p, s}$, we blow up only components of $\left(\operatorname{inv}=\operatorname{inv}_{p, s}\right)$ that are disjoint from $\Sigma_{p}(X)$ (Remark 4.1 still applies).

Example 5.2. Consider $X=(x(x+y z)=0), E=\emptyset$. The standard resolution algorithm A first blows up $C_{0}=\{0\}$ and afterwards gives:

\begin{tabular}{c|c|c|c|c}
\hline year $j$ & chart & marked ideal $\underline{\underline{I}}^{0}$ & $\operatorname{inv}(0)$ & centre $C_{j}$ \\
\hline \hline 1 & $(x y, y, y z)$ & $(x y(x+y z), 1)$ & $(2,0,1,1,1,0, \infty)$ & $\{0\}$ \\
\hline 2 & $(x y, y, y z)$ & $\left(x y^{2}(x+y z), 1\right)$ & $(2,0,1,0, \infty)$ & $(x=z=0)$ \\
\hline
\end{tabular}

Note that the centre $C_{2}$ includes snc points. In year one, $\operatorname{inv}(0)=\operatorname{inv}_{2, s}, s=(0,1)$, but Algorithm $\mathrm{C}$ (above) also blows up $C_{1}=\{0\}$. In year two, $\operatorname{inv}(0)=\operatorname{inv}_{2,0}$; Algorithm C (and B) continues with a cleaning blowing-up, center $(x=y=0)$, after which we have snc over the chart shown.

The variant of Algorithm C in Remark 5.1, however, cleans up with centre $(x=$ $y=0$ ) in year one, providing the following instead of the previous table. (Here we already have snc in the year-two chart shown.)

\begin{tabular}{c|c|c|c|c}
\hline year $j$ & chart & marked ideal $\underline{\underline{I}}^{0}$ & $\operatorname{inv}(0)$ & centre $C_{j}$ \\
\hline \hline 1 & $(x y, y, y z)$ & $(x y(x+y z), 1)$ & $(2,0,1,1,1,0, \infty)$ & $(x=y=0)$ \\
\hline 2 & $(x y, y, z)$ & $\left(x y^{2}(x+z), 1\right)$ & $(2,0,1,1,1,0, \infty)$ & \\
\hline
\end{tabular}

As described above, Algorithm $\mathrm{C}$ (as well as its variant) requires that inv, suitably modified, remains well defined after a cleaning blowing-up. As an illustration, the invariant in year two of the preceding table (i.e., after the cleaning blowing-up with centre $C_{1}$ ) is computed as follows (using the pattern of Example 2.2):

\begin{tabular}{c|c|c|c|c}
\hline $\begin{array}{c}\text { codim } \\
i\end{array}$ & $\begin{array}{c}\text { marked ideal } \\
\underline{\underline{I}}^{i}\end{array}$ & $\begin{array}{c}\text { companion ideal } \\
\underline{\mathcal{J}}^{i}=\underline{\mathcal{G}}\left(\underline{\mathcal{I}}^{i}\right)\end{array}$ & $\begin{array}{c}\text { maximal } \\
\text { contact }\end{array}$ & $\begin{array}{c}\text { boundary } \\
E^{i+1}\end{array}$ \\
\hline \hline 0 & $\left(x y^{2}(x+z), 1\right)$ & $(x(x+z), 2)$ & $(x=0)$ & \\
\hline 1 & $(z, 1)$ & $(z, 1)$ & $(x=z=0)$ & $(y=0)$ \\
\hline 2 & $(y, 1)$ & $(y, 1)$ & $(x=y=z=0)$ & \\
\hline 3 & $(0)$ & & & \\
\hline
\end{tabular}

The cleaning blowing-up with centre $C_{1}$ moves the exceptional divisor $(y=0)$ of year one away from the year-two chart given by $(x y, y, z)$. In the latter, $(y=0)$ is the new exceptional divisor, but is treated simply as a relabeling of the old $(y=0)$; 
i.e., it is still counted as a member of $E^{2}$. The value of inv(0) in year two here is unchanged from year one, as remarked in the proof of Lemma 4.4

\section{REFERENCES}

[1] Edward Bierstone and Pierre D. Milman, Canonical desingularization in characteristic zero by blowing up the maximum strata of a local invariant, Invent. Math. 128 (1997), no. 2, 207-302, DOI 10.1007/s002220050141. MR1440306 (98e:14010)

[2] Edward Bierstone and Pierre D. Milman, Functoriality in resolution of singularities, Publ. Res. Inst. Math. Sci. 44 (2008), no. 2, 609-639, DOI 10.2977/prims/1210167338. MR2426359 (2009d:14010)

[3] Edward Bierstone and Pierre D. Milman, Resolution except for minimal singularities I, Adv. Math. 231 (2012), no. 5, 3022-3053, DOI 10.1016/j.aim.2012.08.002. MR.2970472

[4] Edward Bierstone and Franklin Vera Pacheco, Resolution of singularities of pairs preserving semi-simple normal crossings, Rev. R. Acad. Cienc. Exactas Fís. Nat. Ser. A Mat. RACSAM 107 (2013), no. 1, 159-188. MR.3031268

[5] János Kollár, Semi log resolutions, preprint arXiv:0812.3592v1 [math.AG] (2008), 10 pages.

[6] Endre Szabó, Divisorial log terminal singularities, J. Math. Sci. Univ. Tokyo 1 (1994), no. 3, 631-639. MR.1322695 (96f:14019)

The Fields Institute, 222 College Street, Toronto, Ontario, Canada M5T 3J1 - and - Department of Mathematics, University of Toronto, 40 St. George Street, Toronto, Ontario, Canada M5S 2E4

E-mail address: bierston@math.toronto.edu

Department of Mathematics, University of Toronto, 40 St. George Street, Toronto, Ontario, Canada M5S 2E4

Current address: Department of Mathematics, Cornell University, 310 Malott Hall, Ithaca, New York 14853

E-mail address: smd322@cornell.edu

Department of Mathematics, University of Toronto, 40 St. George Street, Toronto, Ontario, Canada M5S 2E4

E-mail address: milman@math.toronto.edu

The Fields Institute, 222 College Street, Toronto, Ontario, Canada M5T 3J1

E-mail address: franklin.vp@gmail.com 